\title{
Eficiência energética aplicada ao consumo de eletricidade: Um estudo de revisão
}

\section{bibliográfica}

\author{
Energy efficiency applied to electricity consumption: A bibliographic review study \\ Eficiencia energética aplicada al consumo eléctrico: Estudio de revisión bibliográfica
}

Recebido: 19/03/2021 | Revisado: 26/03/2021 | Aceito: 31/03/2021 | Publicado: 10/04/2021

Juliana dos Santos Costa

ORCID: https://orcid.org/0000-0001-6807-6210 Instituto Federal de Educação, Ciência e Tecnologia Fluminense, Brasil E-mail: julianadossantosc@gmail.com

Luiz Maurício de Andrade Junior

ORCID: https://orcid.org/0000-0001-8335-7416 Instituto Federal de Educação, Ciência e Tecnologia Fluminense, Brasil

E-mail: 1mjunior@iff.edu.br

\begin{abstract}
Resumo
A crescente demanda por eletricidade vem estimulando estudos e ações não somente para o aumento da capacidade de geração de usinas, mas também o uso racional dessa importante forma de energia. Com isso, é notório o crescimento no número de publicações relacionadas ao tema, gerando assim um número demasiado de trabalhos, o que torna a filtragem e análise complexa. Nesse contexto, a revisão bibliográfica é uma importante ferramenta para verificação quantitativa e qualitativa dos estudos publicados nos diversos periódicos. Diante do exposto, o presente artigo tem como objetivo realizar uma análise bibliométrica da produção científica brasileira sobre a eficiência energética aplicada ao consumo de eletricidade. A base de dados Scopus foi utilizada para a busca dos artigos relacionados ao tema. Foram encontrados um total de 66 trabalhos no período de 2004 a 2020, no entanto, após uma leitura dinâmica verificou-se que apenas 37 desses discorrem efetivamente sobre a eficiência energética atrelada ao consumo de eletricidade. Desses, 83,8\% correspondem a trabalhos de estudo de caso, 10,8\% as políticas públicas e 5,4\% foram estudos de revisão bibliográfica. A análise permitiu constatar que grande parte dos artigos de estudo de caso foram aplicados ao setor industrial (45,2\%), especialmente aos sistemas motrizes. Além disso, observou-se que mesmo o setor residencial sendo um dos principais consumidores de energia, há um número de publicações pouco expressivo, indicando assim uma lacuna a ser preenchida.
\end{abstract}

Palavras-chave: Eficiência energética; Energia elétrica; Bibliometria.

\begin{abstract}
The growing demand for electricity has been stimulating studies and actions not only to increase the generation capacity of plants, but also the rational use of this important form of energy. As a result, there is a notable growth in the number of publications related to the theme, thus generating too many works, which makes filtering and analysis complex. In this context, the bibliographic review is an important tool for quantitative and qualitative verification of studies published in different journals. Given the above, this article aims to perform a bibliometric analysis of Brazilian scientific production on energy efficiency applied to electricity consumption. The Scopus database was used to search for articles related to the topic. A total of 66 papers were found in the period from 2004 to 2020, howe ver, after a dynamic reading it was found that only 37 of them effectively talk about energy efficiency linked to electricity consumption. Of these, $83.8 \%$ correspond to case study works, $10.8 \%$ to public policies and $5.4 \%$ were literature review studies. The analysis showed that most of the case study articles were applied to the industrial sector (45.2\%), especially to the driving systems. In addition, it was observed that even though the residential sector is one of the main consumers of energy, there is a small number of publications, thus indicating a gap to be filled.
\end{abstract}

Keywords: Energy efficiency; Electricity; Bibliometrics.

\section{Resumen}

La creciente demanda de electricidad ha estado estimulando estudios y acciones no solo para aumentar la capacidad de generación de las plantas, sino también el uso racional de esta importante forma de energía. Como resultado, se observa un notable crecimiento en el número de publicaciones relacionadas con la temática, generando así demasiados trabajos, lo que hace complejo el filtrado y análisis. En este contexto, la revisión bibliográfica es una herramienta importante para la verificación cuantitativa y cualitativa de estudios publicados en diferentes revistas. Dado lo anterior, este artículo tiene como objetivo realizar un análisis bibliométrico de la producción científica brasileña sobre eficiencia energética aplicada al consumo eléctrico. Se utilizó la base de datos Scopus para buscar artículos relacionados con el tema. Se encontraron un total de 66 artículos en el período de 2004 a 2020, sin embargo, luego de 
una lectura dinámica se encontró que solo 37 de ellos hablan efectivamente de eficiencia energética conectada al consumo eléctrico. De estos, el $83,8 \%$ corresponden a trabajos de estudios de caso, el 10,8\% a políticas públicas y el $5,4 \%$ a estudios de revisión de la literatura. El análisis mostró que la mayoría de los artículos de los estudios de caso se aplicaron al sector industrial (45,2\%), especialmente a los sistemas de conducción. Además, se observó que a pesar de que el sector residencial es uno de los principales consumidores de energía, existe un número reducido de publicaciones, lo que indica un vacío por llenar.

Palabras clave: Eficiencia energetica; Energia electrica; Bibliometría.

\section{Introdução}

No atual cenário energético mundial, o consumo de energia é um importante indicador de desenvolvimento para os países. No geral, nações desenvolvidas aumentam sua demanda por energia à medida que sua economia se desenvolve (Souza et al., 2020). Segundo a International Energy Agency [IEA] (2019), só no ano de 2018 a demanda mundial por eletricidade apresentou um aumento de $4,1 \%$ em relação ao ano anterior.

O Brasil, sendo um país em desenvolvimento, segue a mesma tendência dos países já desenvolvidos, na qual espera-se que haja um crescimento no consumo de eletricidade proporcional ao desenvolvimento de sua economia e surgimento de novas tecnologias (Oliveira et al., 2017). Segundo a Empresa de Pesquisa Energética (EPE, 2020), no ano de 2019, o Brasil apresentou uma progressão de 1,3\% na demanda final por energia elétrica, sendo os setores que mais colaboraram para essa progressão, em termos de valores absolutos, o comercial $(+4,5)$, o residencial $(+3,5 \%)$, o energético $(+4,1)$ e o público $(+2,1)$.

Desse modo, se mostra necessário a implementação de medidas que possibilitem não só o aumento da oferta de energia por meio de fontes renováveis, mas também o seu uso de maneira racional e eficiente (Leite et al., 2019).

De acordo com Souza et al. (2019) a eficiência energética pode ser definida como a obtenção de um mesmo serviço energético, utilizando menos energia durante o processo. Nesse contexto, é possível proporcionar o uso racional e eficiente da energia em todas as etapas do processo, desde sua obtenção em forma primária até o consumo final.

A eficiência energética se mostra como uma ferramenta de relevante impacto e baixo custo para redução dos gastos com energia, podendo alcançar aproximadamente $20 \%$ de economia quando comparada à aquisição de novas fontes de geração (Silva, Oliveira \& Tostes, 2017). Dessa forma, um sistema de gestão energética implementa, constantemente, métodos técnicoorganizacionais que buscam oportunidades de conservação e utilização eficiente da energia nas instalações, tendo como objetivo a redução dos custos (Batlle et al., 2020).

A implantação de políticas públicas governamentais é um dos fatores que podem agilizar a adoção de medidas de eficiência energética pela população. No entanto, para que possam causar verdadeiro impacto na sociedade, essas políticas devem ser propostas juntamente com incentivos fiscais e financeiros (Fabbriani \& Calili, 2018). Ademais, a tarefa de avaliar o resultado dessas medidas na melhoria da eficiência energética é um obstáculo a ser vencido, já que se faz necessário a comparação entre demanda de energia antes e após a implementação das ações (Bortoni et al., 2020).

Como exposto, a eficiência energética tem se tornado uma ferramenta eficaz para mitigar problemas relacionados com a crescente demanda por eletricidade e promover o uso racional da energia gerada. Diante disso, este artigo se propôs a realizar uma revisão bibliométrica da produção científica brasileira sobre eficiência energética aplicada ao consumo de eletricidade. Para tanto, foram utilizados artigos indexados na base de dados Scopus.

\section{Marcos Regulatórios da Eficiência Energética}

O Programa Conserve, criado em 1981, foi um incentivo governamental brasileiro que buscou dar ampla visualização à utilização de medidas de eficiência energética. Tal programa tinha como objetivo estimular a conservação de energia na indústria, a partir da eficientização de produtos/processos e da transição de insumos importados por alternativas nacionais. Em seguida, no ano de 1982, foi aprovado o Programa de Mobilização Energética (PME), que consistia em um conjunto de 
procedimentos guiados para promover a racionalização do consumo de eletricidade e substituição progressiva de fontes de energia derivadas do petróleo (Ministério de Minas e Energia [MME], 2011).

No ano de 1985, os Ministérios de Minas e Energia e da Indústria e do Comércio, por intermédio da Portaria Interdisciplinar $\mathrm{n}^{\circ}$ 1877, instituíram o Programa Nacional de Conservação de Energia Elétrica (Procel). Este tinha como finalidade a integração das medidas de conservação de eletricidade no país, buscando a maximização de seus resultados. Dentre uma de suas ações encontra-se a fundação do Programa Brasileiro de Etiquetagem (PBE) (Brasil, 1985).

Em 1991, com o decreto presidencial de 18 de julho deste ano, foi criado o Programa Nacional da Racionalização do Uso de Derivados do Petróleo e do Gás Natural (CONPET). O programa tinha como objetivo promover medidas para racionalização do consumo de fontes não renováveis no país (Brasil, 1991).

No ano de 1997, com a Lei n 9.478 foi instituída a Política Energética Nacional (PEN), o Conselho Nacional de Política Energética e a Agência Nacional de Petróleo. A partir desta lei foram estabelecidos os princípios e objetivos da Política Energética Nacional, sendo um deles a proteção do meio ambiente e conservação de energia. Com essa lei foram instituídas também as atribuições de cada entidade criada (Brasil, 1997).

Em 2000, foi promulgada a Lei $n^{\circ}$ 9.991, que discorria sobre a obrigação das empresas concessionárias, permissionárias e autorizadas do ramo de eletricidade investirem em pesquisas/desenvolvimento e eficiência energética (Brasil, 2000).

No ano de 2001, com a Lei n ${ }^{\circ}$ 10.295, amplamente conhecida como Lei da Eficiência Energética, foi criada a Política Nacional de Conservação e Uso Racional de Energia. Essa lei tinha como objetivo a eficientização na destinação de insumos energéticos e a preservação ambiental (Brasil, 2001a). No mesmo ano, por intermédio do Decreto $\mathrm{n}^{\circ} 4.059 / 2001$ foram estabelecidos os indicadores máximos ou mínimos de eficiência energética de equipamentos fabricados ou comercializados no Brasil e das edificações a serem construídas (Brasil, 2001b). O Decreto $\mathrm{n}^{\circ} 4.059 / 2001$ foi revogado por meio do Decreto $\mathrm{n}^{\circ}$ 9.864/2019, que regulamenta a Lei da Eficiência Energética e discorre sobre Política Nacional de Conservação e Uso Racional de Energia e o Comitê Gestor de Indicadores e Níveis de Eficiência Energética (Brasil, 2019).

O Plano Nacional de Energia (PNE) 2030, publicado em 2007, foi o primeiro estudo elaborado pelo governo brasileiro na esfera de planejamento energético a longo prazo. Esse plano teve o intuito de apresentar o potencial do país para a implementação de medidas de eficiência energética, estratégias para a ampliação da oferta de energia de maneira sustentável, além de prover insumos para a elaboração de política energética (Ministério de Minas e Energia [MME], 2007).

No ano de 2009, o PBE sofreu alterações. Neste ano foi divulgado o programa de etiquetagem de edifícios no Brasil, o qual abrangia prédios comerciais, públicos e de serviços. No ano seguinte os edifícios residenciais foram incluídos no programa (Altoé et al.,2017).

O Plano Nacional de Eficiência Energética (PNEf), publicado em 2011, teve como objetivo apresentar um conjunto de medidas para a promoção da eficiência energética nos diversos setores da economia, identificando meios para obtenção de recursos energéticos, aprimoramento do marco legal e conscientização da população para o consumo consciente (MME,2011).

\section{Metodologia}

A bibliometria é uma das principais ferramentas de pesquisa que utiliza métodos estatísticos para analisar a produção acadêmica contida em diversos tipos de documentos (Cabeza et al., 2020). É comumente utilizada para mensurar quantitativamente a evolução do conhecimento científico e tecnológico sobre um determinado assunto, além de elucidar as tendências de pesquisa de diversas áreas (Camarasa et al., 2019; Mao et al., 2015).

Um estudo de revisão bibliométrica leva em consideração índices de produção científica que além de ilustrar as principais características dos artigos, também auxilia na compreensão geral do tema estudado. Dentre esses índices se 
encontram a distribuição temporal e geográfica do assunto, os pesquisadores, periódicos e áreas de estudo com maior número de publicações, entre outros (Visentin et al., 2019; Visentin et al., 2020; Wang et al., 2017).

Para a realização da análise bibliométrica deste estudo, foram utilizados artigos indexados na base de dados Scopus, com o objetivo de avaliar a produção científica brasileira sobre o tema eficiência energética no consumo de eletricidade. A Scopus foi escolhida por ser uma abrangente base de dados, que possui mais de 22.000 títulos, provenientes de mais de 5.000 editores de todo o mundo. Além disso, compreende diversas áreas de estudo, tais como, ciências, tecnologia, ciências sociais, medicina, artes e humanidades (Scopus, 2020).

Como critério de seleção dos artigos foi utilizada a expressão idiomática (TITLE-ABS-KEY ("energy efficiency" AND "electricity") AND TITLE-ABS-KEY (“buildings" OR “industries")) AND (LIMIT-TO (AFFILCOUNTRY, "Brazil”)) AND (LIMIT-TO (DOCTYPE, “ar”)), que resultou em um total de 66 artigos, no período de 2004 a 2020.

Visando uma correta análise dos dados e seleção apenas dos documentos alinhados ao tema da pesquisa, se fez necessário uma leitura dinâmica de todos os artigos encontrados. Desse modo, 29 artigos foram desconsiderados, sendo 24 deles por terem relação com a eficiência energética, mas não estarem atrelados ao consumo de eletricidade, e 5 deles por não serem aplicados no Brasil, já que a plataforma Scopus considera como artigos limitados ao Brasil todos aqueles em que pelo menos um dos autores tenha nacionalidade brasileira. Isto posto, 36 artigos foram considerados neste trabalho.

Com base nos artigos selecionados, realizou-se uma segmentação quantitativa dos dados, verificando a evolução das publicações ao longo dos anos, os autores e periódicos que mais publicaram, os artigos mais citados e as áreas temáticas com maior número de publicações.

\section{Resultados e Discussão}

\subsection{Publicações por Ano}

Na Figura 1 é apresentada a evolução ao longo dos anos dos artigos considerados para o estudo.

Figura 1 - Número de publicações ao longo dos anos.

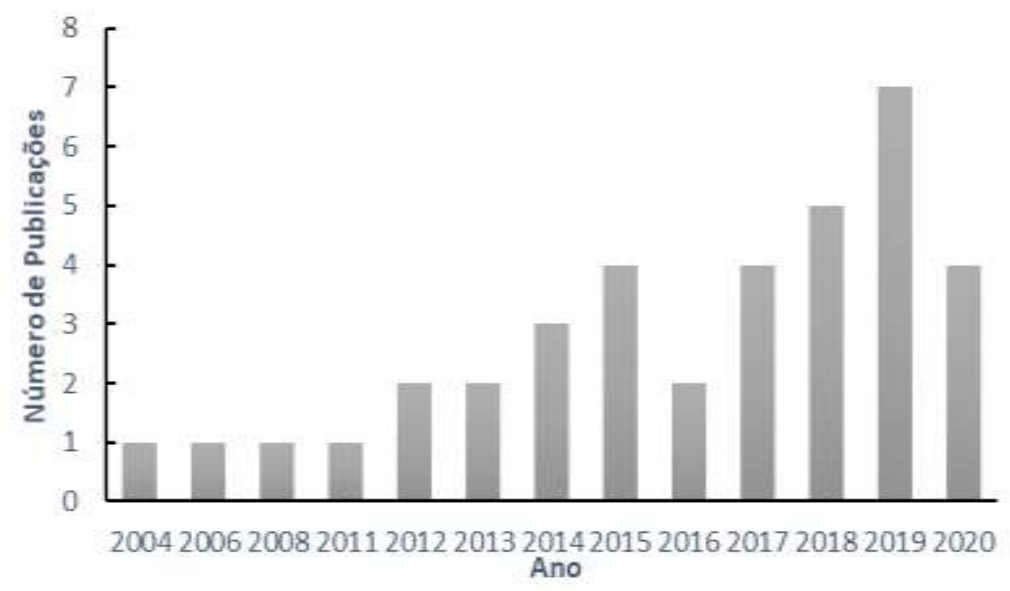

Fonte: Autores (2021).

O primeiro artigo publicado sobre o tema, na base de dados Scopus, é datado de 2004 e intitulado "The Brazilian energy crisis and a study to support building efficiency legislation". Neste artigo Rosa e Lomardo (2004) apresentaram um estudo de caso paramétrico de um edifício comercial na cidade do Rio de Janeiro. Para isso, realizaram simulações para avaliar as variações da relação janela-parede, considerando a presença e ausência de luz natural, além de vidros e tonalidades interiores diferentes. 
É possível observar pela Figura 1 que apesar do assunto ter sido abordado pela primeira vez no ano de 2004, até o ano de 2011 houve um pequeno número de publicações (um artigo por ano). Em 2011, foi criado o PNEf, no qual são apresentadas um conjunto de ações e medidas para conservação da energia elétrica em diversas áreas, como indústria, edificações, transportes, educação, iluminação pública, aquecimento solar de água, entre outros (Altoé et al.,2017; Calili et al. 2014). Acredita-se que o lançamento do PNEf influenciou novos estudos (publicações acadêmicas) relacionados ao tema. Após o ano de 2011, houve um aumento no número de artigos publicados, sendo o ápice no ano de 2019 (sete artigos).

\subsection{Número de Publicações por periódico}

O Quadro 1 apresenta a relação dos dez primeiros periódicos que mais publicaram sobre o tema deste estudo na base de dados Scopus.

Quadro 1 - Número de publicações por periódico.

\begin{tabular}{|l|c|}
\hline \multicolumn{1}{|c|}{ Periódico } & $\mathbf{N}^{\mathbf{0}}$ de Publicações \\
\hline Energy & 4 \\
\hline Energy and Buildings & 4 \\
\hline Espacios & 3 \\
\hline Renewable Energy and Power Quality Journal & 3 \\
\hline Energy for Sustainable Development & 2 \\
\hline Energy Policy & 2 \\
\hline IEEE Latin America Transactions & 2 \\
\hline Journal of Cleaner Production & 2 \\
\hline Acta Scientiarum & 1 \\
\hline Applied Sciences & 1 \\
\hline
\end{tabular}

Fonte: Autores (2021).

Energy e Energy and Buildings, foram os periódicos que mais publicaram sobre o assunto, ambos com quatro publicações. Energy é um jornal internacional multidisciplinar que aborda temas de engenharia e pesquisa energética. Energy and Buildings é uma revista internacional que aborda a redução do uso energia em edifícios e a melhora da qualidade do ambiente interno.

\subsection{Número de Publicações por autor}

O Quadro 2 apresenta uma listagem dos dez primeiros autores que mais publicaram sobre o tema na base de dados Scopus.

Quadro 2 - Número de publicações por autor.

\begin{tabular}{|l|c|}
\hline \multicolumn{1}{|c|}{ Autor } & $\mathbf{N}^{\mathbf{0}}$ de Publicações \\
\hline Souza, R.C. & 4 \\
\hline Calili, R.F & 2 \\
\hline Oliveira, F. L. C. & 2 \\
\hline Lafay, J.M.S. & 2 \\
\hline Carlo, J. & 2 \\
\hline Rocca, G.A.D. & 2 \\
\hline Bajay, S.V. & 2 \\
\hline Stefenon, S.F. & 2 \\
\hline Portela, T.T. & 2 \\
\hline Da Silva, Felipe L.C. & 1 \\
\hline
\end{tabular}

Fonte: Autores (2021). 
O autor Souza foi o que mais publicou sobre o tema, com quatro artigos publicados. O autor é vinculado à Pontifícia Universidade Católica do Rio de Janeiro (PUC-Rio) desde 1972, integra o departamento de engenharia industrial desde 2017 e é membro da área de concentração gerência de produção (linha de pesquisa: análises de séries temporais).

\subsection{Número Citações por Artigo}

O Quadro 3 demonstra uma listagem com os 3 primeiros artigos mais citados na plataforma Scopus.

Quadro 3 - Artigos mais citados.

\begin{tabular}{|l|c|}
\hline \multicolumn{1}{|c|}{ Título } & $\mathbf{N}^{\mathbf{0}}$ Citações \\
\hline $\begin{array}{l}\text { Development of envelope efficiency labels for commercial buildings: } \\
\text { Effect of different variables on electricity consumption }\end{array}$ & 48 \\
\hline $\begin{array}{l}\text { Technical and economic evaluation of thin-film CdTe building- } \\
\text { integrated photovoltaics (BIPV) replacing façade and rooftop } \\
\text { materials in office buildings in a warm and sunny climate }\end{array}$ & 36 \\
\hline $\begin{array}{l}\text { Energy, exergy, sustainability, and emission analysis of industrial air } \\
\text { compressors }\end{array}$ & 26 \\
\hline
\end{tabular}

Fonte: Autores (2021).

$\mathrm{O}$ artigo intitulado "Development of envelope efficiency labels for commercial buildings: Effect of different variables on electricity consumption" foi o mais citado nos termos desta pesquisa, com quarenta e oito citações. Tal fato, denota a relevância desse artigo para o tema eficiência energética. Neste artigo Carlo e Lamberts (2008) descreveram os critérios necessários para obtenção das etiquetas de eficiência do invólucro para prédios comerciais. Nesse contexto, os autores desenvolveram uma equação de regressão linear multivariável para estimar o indicador de consumo de eletricidade.

Com relação a segunda publicação mais citada, Sorgato e Rüther (2018) avaliaram o desempenho energético de um prédio de escritórios, considerando a integração de um sistema fotovoltaico com módulos de filmes finos de telureto de cádmio. Com esse intuito, os autores realizaram simulação para seis cidades brasileiras distintas, além de verificarem a viabilidade econômica de substituição de materiais da fachada tradicional pelos módulos fotovoltaicos.

Quanto à terceira publicação mais citada, Mascarenhas et al. (2019) realizaram uma ampla análise do desempenho energético, eficiência exergética, emissão de $\mathrm{CO} 2$, sustentabilidade e viabilidade econômica de um sistema de ar comprimido de uma indústria manufatureira. Para tornar cada um desses parâmetros um valor palpável, os autores realizaram diversos cálculos baseados em outras bibliografias e, além disso, utilizaram o software de simulação RETScreen para a realização da análise econômica.

\subsection{Número de Publicações por Área Temática}

O Quadro 4 expõe uma listagem com todas as áreas temáticas que publicaram sobre o tema na base de dados Scopus. 
Quadro 4 - Número de publicações por área temática.

\begin{tabular}{|l|c|}
\hline \multicolumn{1}{|c|}{ Áreas de Estudo } & $\mathbf{N}^{\mathbf{0}}$ de Publicações \\
\hline Engineering & 22 \\
\hline Energy & 17 \\
\hline Environmental Science & 13 \\
\hline Social Sciences & 2 \\
\hline Computer Science & 4 \\
\hline Mathematics & 2 \\
\hline Physics and Astronomy & 2 \\
\hline Chemistry & 1 \\
\hline Earth and Planetary Sciences & 2 \\
\hline Business, Management and Accounting & 2 \\
\hline Materials Science & 2 \\
\hline Chemical Engineering & 1 \\
\hline Decision Sciences & 3 \\
\hline
\end{tabular}

Fonte: Autores (2021).

Os artigos selecionados para o estudo se enquadraram em 13 áreas temáticas distintas, demonstrando que o tema abordado interage com diversas áreas de conhecimento. Dentre as áreas temáticas que obtiveram o maior número de publicações destacam-se as áreas de engenharia, energia e ciência ambiental com 22, 17 e 13 publicações, respectivamente. Vale ressaltar que, cada um dos 37 artigos selecionados para análise, está atrelado a mais de uma área temática simultaneamente.

\subsection{Número de artigos por tipologia de publicação}

A Figura 2 ilustra a segmentação dos 37 artigos selecionados para estudo de acordo com sua tipologia.

Figura 2 - Segmentação dos artigos selecionados de acordo com sua tipologia.

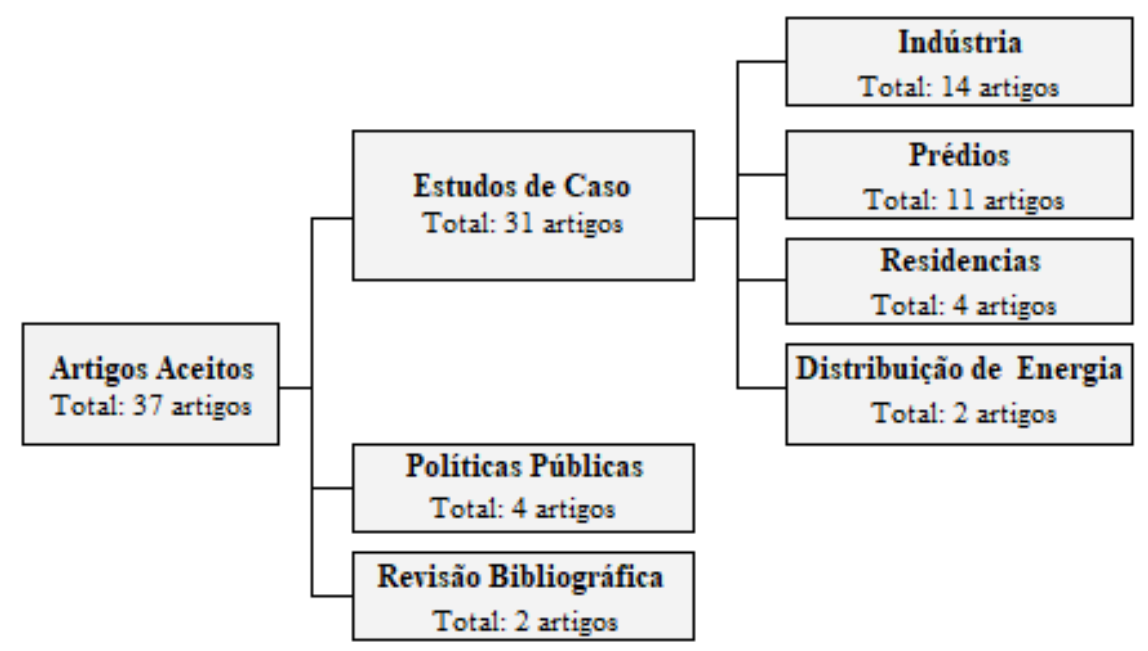

Fonte: Autores (2021).

Observando a Figura 2, verifica-se que 31 artigos (83,8\%) são desenvolvidos na tipologia de estudos de caso, 4 artigos (10,8\%) análises de políticas públicas e 2 artigos (5,5\%) análises bibliográficas.

É possível concluir que grande parte dos trabalhos selecionados são estudos de caso aplicados à indústria. Segundo a EPE (2020), o setor industrial é responsável pelo maior consumo de eletricidade do Brasil, representando uma parcela de $35,9 \%$ do consumo total. Apesar disso, esse setor apresenta uma das maiores tarifas com megawatt-hora, o que prejudica sua 
competitividade tanto no mercado nacional como internacional. Nesse contexto, há uma crescente busca por alternativas que proporcionem a eficiência energética na indústria, visando a redução dos custos, a otimização do consumo, a diminuição dos impactos ambientais e consequentemente o aumento da competitividade (Sola e Mota, 2015; Viana, 2019; Zanardo et al., 2018).

$\mathrm{Na}$ indústria os sistemas motrizes representam, em média, $24 \%$ do consumo total de energia elétrica consumida no Brasil (Segundo et al., 2018). Considerando as crescentes demandas de energia e sustentabilidade industrial, frente a competitividade do setor, máquinas e processos mais eficientes são fundamentais para o segmento. Essa necessidade foi refletida no levantamento bibliométrico realizado neste artigo, pois do total dos estudos de caso aplicados à indústria, 40\% dos trabalhos tinham relação direta com os motores elétricos. De acordo com Segundo et al. (2018), a correta adequação da força motriz da planta industrial representa uma das estratégias para a eficientização do sistema. Tal estratégia possui potencial de mais de 50\% de redução no consumo de eletricidade numa indústria de laticínios, onde o estudo foi implementado. Pelegrino et al. (2019) em estudo aplicado aos centros de usinagem, apontam que a operação inteligente das máquinas e ferramentas envolvidas nos processos de produção, é uma importante estratégia para a redução da energia consumida, adaptando assim a demanda de energia às exigências no momento da produção.

O processo de tomada de decisão constitui-se um grande desafio para os gestores e uma importante tarefa para o crescimento e aumento de competitividade de uma indústria no mercado onde está inserida. Silva et al. (2018), Silva et al. (2019) e Zanardo et al. (2018) sugeriram modelos, combinando uma ou mais metodologias para diagnóstico do nível de eficiência energética e elaboração de cenários de consumos. O método Bottom-up foi aplicado nos trabalhos de Silva et al. (2018) e Silva et al. (2019), a fim de prever o consumo de eletricidade a longo prazo de um setor industrial específico (celulose e papel), considerando cenários de eficiência energética. Silva et al. (2019) enfatizam que construir uma abordagem Buttom-up no Brasil, país em desenvolvimento, é muito mais difícil, pois o fato do modelo requerer muitos dados por parte das indústrias, torna o processo ainda mais desafiador. Tal dificuldade poderá ser reduzida com o advento da indústria 4.0, já que a digitalização e a conectividade são requisitos básicos do conceito.

Os prédios públicos e comerciais representam juntos uma parcela de $25,9 \%$ do consumo de eletricidade do Brasil (EPE, 2020). Desse modo, a eficiência energética destes edifícios está ganhando cada vez mais espaço no cenário nacional, visto que apresentam inúmeras oportunidades de otimização do consumo de energia elétrica, tais como o aprimoramento do isolamento da envoltória e a adoção de condicionadores de ar e equipamentos de alta eficiência (Batlle et al., 2020).

Este trabalho identificou 11 artigos relacionados à eficiência energética nos edifícios. Sendo 45\% destes aplicados a edifícios comerciais e 36\% a prédios públicos, especialmente voltados para o ramo educacional (universidades, bibliotecas, entre outros). Sorgato e Rüther (2018) apontam que o grande desafio desse tipo de edificação no Brasil tem sido a redução do consumo de eletricidade, e paralelamente, o aumento da autogeração por meio de sistemas descentralizados. Verifica-se, nesse contexto, um incremento na implementação da geração fotovoltaica $(\mathrm{FV})$, pois esta vem ganhando força nos últimos anos, principalmente em função da queda dos custos de aquisição dos sistemas. Além do Brasil possuir fatores climáticos propícios para a implementação da tecnologia $\mathrm{FV}$, outra vantagem é a sinergia entre geração e o pico de consumo de energia elétrica nessas edificações, devido à demanda de energia em função do uso dos aparelhos de ar condicionado.

Nos prédios públicos, principalmente os voltados para atividades de educação, como uma instituição de ensino superior (IES), aspectos relacionados ao sistema de ar condicionado, sistema de iluminação e fatores construtivos possuem significativos impactos no consumo de energia (Batlle et al., 2020). Segundo o Programa de Nacional de Conservação de Energia Elétrica, estima-se que o sistema de ar condicionado responde por $48 \%$ do consumo de eletricidade, enquanto o sistema de iluminação é responsável por $23 \%$ do consumo total. Gamarra et al. (2020) em seu estudo de caso, utilizaram o software Energy Plus para modelagem e simulação de retrofit dos condicionadores de ar de uma biblioteca, além da instalação 
de películas em janelas para o controle de temperatura interna. Após validação, a simulação no software apontou um potencial de economia anual de 42,08\% com ar condicionado, por meio de um sistema Variable Refrigerant Flow (VRF). Enquanto o uso de películas de controle solar apresentou uma economia de 4,59\% anuais. O ponto negativo verificado no estudo foi o elevado tempo para retorno do investimento (payback) para o sistema de refrigeração (14 anos), além da inviabilidade econômica dos filmes solares.

Godinho et al. (2019) realizaram um estudo de eficiência energética e viabilidade econômica voltado à iluminação do Centro de Ciências Exatas e Tecnologia da UNIPLAC. No referido trabalho os autores usaram uma abordagem quantitativa a fim de argumentar as hipóteses e cenários. Concluíram que o potencial de redução do consumo de energia pelo sistema de iluminação é de quase 50\% quando as lâmpadas fluorescentes forem substituídas por lâmpadas LED, mantendo o mesmo nível de iluminância (lux), porém com potência elétrica reduzida. Outras características mostram que as lâmpadas LED são mais eficientes que as lâmpadas fluorescentes, como o fluxo luminoso, eficiência luminosa e vida útil. Dado o estudo de viabilidade econômica, o tempo do payback foi de aproximadamente 3 anos, sendo considerado viável e interessante pelos autores.

Para além de retrofit de sistemas e máquinas, Silva et al. (2016) evidência que a Gestão Ambiental em universidades é primordial não somente para trazer benefícios financeiros (aumento de produtividade, redução no consumo de energia, água e materiais), mas também permite adequação à legislação ambiental, aumento da confiabilidade do sistema, melhorias no conforto e na imagem da instituição perante à sociedade. Os autores afirmam ainda que, num sistema de gestão, é importante estabelecer objetivos e metas que sejam específicos e mensuráveis, a fim de validar o Plano de Eficiência Energética (PEE) com bases em protocolos internacionais de Medição e Verificação (M\&V).

O setor residencial é o segundo maior consumidor de energia elétrica do Brasil, representando uma parcela de 26,1\% do consumo total (EPE, 2020). No entanto, percebe-se a partir da Figura 2, que este setor possui um número incipiente de publicações, demonstrando que, nos termos desta pesquisa, existe uma lacuna no desenvolvimento de estudos voltados para esse segmento. Segundo Oliveira et al. (2017), o setor residencial apresentou um crescimento no consumo de eletricidade na última década, devido ao aumento do poder aquisitivo da população e busca incessante por melhores níveis de conforto, tal fato demonstra a necessidade da adoção de medidas de eficiência energética para otimização do consumo nesse setor.

Existe uma tendência de estudos voltados para implementação de sistemas fotovoltaicos como alternativa para melhora da eficiência energética no setor residencial. Leite et al. (2019) propuseram um modelo matemático para avaliar os benefícios econômicos da utilização de sistemas fotovoltaicos integrados a condicionadores de ar. Os resultados demonstraram alta viabilidade econômica, com taxa de retorno interno de 28\% para a cidade do Recife e 22\% para a cidade de São Paulo. Oliveira et al. (2017) apresentaram um protótipo de casa solar com zero energia como ferramenta para o desenvolvimento sustentável. Nesse contexto, verificou-se que a utilização de sistemas FV associados a medidas de eficiência energética, possibilita zerar os gastos relacionados ao consumo de eletricidade.

Quanto aos artigos relacionados ao setor de distribuição de energia elétrica, Heideier et al. (2020) avaliam os impactos econômicos, ambientais, sociais e políticos, percebidos pelas concessionárias de distribuição de eletricidade, a partir da implementação da geração fotovoltaica e medidas de eficiência energética. Costa e Galvis (2016) propuseram uma metodologia para avaliar a curva de carga de concessionárias de energia elétrica realizando o gerenciamento pelo lado da demanda e utilizando como parâmetros a elasticidade do sistema e preço por tempo de uso.

Com relação aos artigos de políticas públicas, Volpi et al. (2006) propuseram políticas para auxiliar a implementação de programas de eficiência energética e adoção de tecnologias de energias renováveis na matriz energética brasileira. Calili et al. (2014) estimaram a economia de energia e redução de emissão de $\mathrm{CO}_{2}$, em 5 anos, com a implementação do PNEf criado em 2011. Fabbriani e Calili (2018) definiram políticas públicas de eficiência energética a serem implementadas no setor 
industrial de alimentos e bebidas. Costa et al. (2019), avaliaram três níveis de agregação (macro, meso, micro) dos Programas de Eficiência Energética (PEE), com um enfoque no âmbito da baixa renda.

Sobre os artigos de revisões bibliográficas, Santos et al. (2013) avaliaram os riscos provenientes da implantação de políticas de eficiência energética em edifícios, baseada estritamente no consumo de energia final. Enquanto, Portela e Lafay (2015) analisaram o panorama nacional da eficiência energética na indústria, assim como estímulos e empecilhos para implementação de um sistema de gestão energética em circuitos de refrigeração.

\section{Considerações Finais}

Este artigo teve como principal objetivo realizar uma análise bibliométrica da produção científica brasileira sobre o tema eficiência energética aplicada ao consumo de eletricidade. A busca permitiu a análise de 36 artigos indexados à base de dados Scopus, elucidando o estado da arte a nível nacional do assunto.

Através da distribuição temporal dos artigos, identificou-se um aumento no número de publicações após o ano de 2011, ano no qual foi instituído o PNEf, que estimulou a adoção de medidas de eficiência energética e, por consequência, publicações de estudos na área. Os periódicos que apresentaram maior número de publicações sobre o assunto foram o Energy e o Energy and Buildings, ambos com 4 artigos. Entre as áreas temáticas de maior publicação destacaram-se as áreas de engenharia, energia e ciência ambiental com 22, 17 e 13 artigos publicados, respectivamente. As outras 11 áreas temáticas com publicações nos termos desta pesquisa, demonstram a ampla interação do tema com outras áreas de conhecimento.

Com base na segmentação dos artigos selecionados, observou-se que grande parte dos trabalhos são estudos de caso aplicados à indústria (45,2\%). Tal fato pode ser atribuído por este setor ser o maior consumidor de energia elétrica a nível nacional. Os estudos apontam ainda que, os motores elétricos exercem importante participação no consumo, possuindo assim um potencial de eficiência em torno de 50\%, quando a força motriz for corretamente implementada na planta industrial.

O setor residencial, mesmo sendo o segundo maior consumidor de eletricidade, apresentou um número incipiente de artigos (12,9\%), evidenciando que, nos termos desta pesquisa, existe uma lacuna no desenvolvimento de estudos voltados para este importante segmento.

Para os edifícios comerciais e públicos os trabalhos apontam as principais estratégias para uma maior eficiência da edificação. Dentre as medidas utilizadas encontram-se, principalmente, o retrofit dos sistemas de iluminação e condicionamento de ar; e a implementação da geração fotovoltaica para redução dos custos com eletricidade, já que este tipo de sistema possui completa sinergia entre geração e consumo.

Este trabalho limitou-se à utilização da base Scopus e ao uso de expressões booleanas para a seleção dos artigos. Embora tenha-se escolhido termos comuns ao tema de pesquisa, é certo que nem todas as expressões tenham sido consideradas, o que acarreta em artigos publicados sobre o tema, porém não incluídos nesta análise. Diante disso, para trabalhos futuros, sugere-se a utilização de outras bases científicas para complementação dos dados aqui já elucidados.

\section{Referências}

Altoé, L., Costa, J. M., Oliveira, D. Filho, Martinez, F. J. R., Ferrarez, A. H. \& Viana, L. A. (2017). Políticas públicas de incentivo à eficiência energética. Estudos Avançados, 31(89), 285-297. https://doi.org/10.1590/s0103-40142017.31890022.

Batlle, E. A. O., Palacio, J. C. E., Lora E. E. S., Reyes, A. M. M., Moreno, M. M., \& Morejón, M. B. (2020). A methodology to estimate baseline energy use and quantify savings in electrical energy consumption in higher education institution buildings: Case study, Federal University of Itajub_a (UNIFEI). Journal of Cleaner Production, 244(1), 1-17. https://doi.org/10.1016/j.jclepro.2019.118551.

Bortoni, E. C., Magalhães, L. P., Nogueira, L. A. H., Bajay, S. V., \& Cassula, A. M. (2020). An assessment of energy efficient motors application by scenarios evaluation. Energy Policy, 140(1), 1-15. https://doi.org/10.1016/j.enpol.2020.111402.

Brasil. (1985). Portaria Interministerial MME/MIC n. 1.877, de 30 de dezembro de 1985. Institui o Programa Nacional de Conservação de Energia Elétrica (Procel) e dá outras providências. Brasília: Diário Oficial da União. http://www.procelinfo.com.br/services/DocumentMan 
agement/FileDownload.EZTSvc.asp?DocumentID=\%7B7650C10ACB32401F8FE38E61390806F7\%7D\&ServiceInstUID=\%7B46764F02-4164-4748-9A41C8E7309F80E1\%7D

Brasil. (1991). Decreto presidencial, de 18 de julho de 1991. Institui o Programa Nacional da Racionalização do Uso dos Derivados do Petróleo e do Gás Natural (Conpet) e dá outras providências. Brasília: Diário Oficial da União, 1991. http://planalto.gov.br/CCIVIL_03/DNN/Anterior\%20a\%202000/1991/Dnn213.htm.

Brasil. (1997). Lei n. 9.478, de 6 de agosto de 1997. Dispõe sobre a política energética nacional, as atividades relativas ao monopólio do petróleo, institui o Conselho Nacional de Política Energética e a Agência Nacional do Petróleo e dá outras providências. Brasília: Diário Oficial da União, 1997. http://www.planalto.gov.br/ccivil_03/leis/19478.htm.

Brasil. (2000). Lei n. 9.991, de 24 de julho de 2000. Dispõe sobre realização de investimentos em pesquisa e desenvolvimento e em eficiência energética por parte das empresas concessionárias, permissionárias e autorizadas do setor de energia elétrica, e dá outras providências. Brasília: Diário Oficial da União, 2000. http://www.planalto.gov.br/ccivil_03/leis/19991.htm.

Brasil. (2001a). Lei n. 10.295, de 17 de outubro de 2001. Dispõe sobre a Política Nacional de Conservação e Uso Racional de Energia e dá outras providências. Brasília: Diário Oficial da União, 2001. http://www.planalto.gov.br/ccivil_03/leis/leis_2001/110295.htm.

Brasil. (2001b). Decreto n. 4.059, de 19 de dezembro de 2001. Regulamenta a Lei ${ }^{\circ}$ 10.295, de 17 de outubro de 2001, que dispõe sobre a Política Nacional de Conservação e Uso Racional de Energia, e dá outras providências. Brasília: Diário Oficial da União, 2001. http://www.planalto.gov.br/ccivil_03/decreto/2001/d4059.htm.

Brasil. (2019). Decreto n. 9.864, de 27 de junho de 2019. Regulamenta a Lei $\mathrm{n}^{\circ}$ 10.295, de 17 de outubro de 2001, que dispõe sobre a Política Nacional de Conservação e Uso Racional de Energia, e dispõe sobre o Comitê Gestor de Indicadores e Níveis de Eficiência Energética. Brasília: Diário Oficial da União, 2019. http://www.planalto.gov.br/ccivil_03/_ato2019-2022/2019/decreto/D9864.htm.

Cabeza, L. F., Chàfer, M., \& Mata E., (2020). Comparative Analysis of Web of Science and Scopus on the Energy Efficiency and Climate Impact of Buildings. Energies, 13(2), 1-24. https://doi.org/10.3390/en13020409.

Calili , R. F., Souza, R. C., Galli, A., Armstrong, M., \& Marcato A. L.. M. (2014). Estimating the cost savings and avoided CO2 emissions in Brazil by implementing energy efficient policies. Energy Policy, 67(1), 4-15. http://dx.doi.org/10.1016/j.enpol.2013.09.071.

Camarasa, C., Nägeli, C., Ostermeyer, Y., Klippel, M., \& Botzler, S. (2019). Diffusion of energy efficiency technologies in European residential buildings: A bibliometric analysis. Energy \& Buildings, 202(1), 2-10. https://doi.org/10.1016/j.enbuild.2019.109339.

Carlo, J., \& Lamberts, R. (2008). Development of envelope efficiency labels for commercial buildings: Effect of different variables on electricity consumption. Energy and Buildings, 40(11), 2002-2008. https://doi.org/10.1016/j.enbuild.2008.05.002.

Costa, A. \& Galvis, J. C, (2016). Demand side management using time of use and elasticity price. IEEE Latin America Transactions. 14(10), 4267-4274. https://doi.org/10.1109/TLA.2016.7786304.

Costa, J., Jr., Diehl, J. C., \& Secomandi, F. (2019). Towards systems-oriented energy solutions: A multilevel analysis of a low-income energy e ciency program in brazil. Sustainability, 11(20), 1-22. http://dx.doi.org/10.3390/su11205799.

Empresa de Pesquisa Energética (2020). Balanço Energético Nacional 2020: Ano base 2019. https://www.epe.gov.br/pt/publicacoes-dadosabertos/publicacoes/balanco-energetico-nacional-2020.

Fabbriani, L., \& Calili, R. (2018). Proposal of energy efficiency policies for food and beverage industry in Brazil. Journal Renewable Sustainable Energy, 10(6), 1-21. https://doi.org/10.1063/1.5002088.

Gamarra, M. G. U., Siqueira, J. A. C, \& Pereira, F. L. (2020). A simulated case study of a library in Brazil to improve energy efficiency. Acta Scientiarum. 42(1), 1-13. https://doi.org/10.4025/actascitechnol.v42i1.47262.

Godinho, J. M., Rocca, G. A. D., Silva, F. C. F., Stefenon, S. F., \& Garcia, A. (2017). Study of energy efficiency and economic feasibility for the lighting of exact and technological science center of UNIPLAC. Espacios. 38(42), 1-23.

Heideier, R., Bajay, S. V., Jannuzzi, G. M., Gomes, R. D. M., Guanais, L., Ribeiro, I., \& Paccola, A. (2020). Impacts of photovoltaic distributed generation and energy efficiency measures on the electricity market of three representative Brazilian distribution utilities. Energy for Sustainable Development. 54(1), 6071. https://doi.org/10.1016/j.esd.2019.10.007.

International Energy Agency (2019). Energy Efficiency 2019. https://www.iea.org/reports/energy-efficiency-2019.

Leite, G. N. P., Weschenfelder, F., Araujo, A. M., Ochoa, A. A. V., Prestrelo, N. F. Neto, \& Kraj, A. (2019). An economic analysis of the integration between air-conditioning and solar photovoltaic systems. Energy Conversion and Management, 185(1), 836-849. https://doi.org/10.1016/j.enconman.2019.02.037.

Mao, G.,Liu, X., Du, H., Zuo, J., \& Wang L. (2015). Way forward for alternative energy research: A bibliometric analysisduring 1994-2013. Renewable and Sustainable Energy Reviews, 48(1), 276-286. http://dx.doi.org/10.1016/j.rser.2015.03.094.

Mascarenhas, J. S., Chowdhury, H., Thirugnanasambandam, M., Chowdhury, T., \& Saidur, R. (2019). Energy, exergy, sustainability, and emission analysis of industrial air compressor. Journal of Cleaner Production, 231(1), 183-195. https://doi.org/10.1016/j.jclepro.2019.05.158.

Ministério de Minas e Energia (2007). Plano Nacional de Energia 2030. https://www.epe.gov.br/pt/publicacoes-dados-abertos/publicacoes/Plano-Nacional-deEnergia-PNE-2030

Ministério de Minas e Energia (2011). Plano Nacional de Eficiência Energética. http://cmsdespoluir.cnt.org.br/Documents/PDFs/Plano_Naci onal_de_Eficixncia_Energxtica_-_PNEf_-_final.pdf. 
Oliveira, C. T., Antonio, F., Burani, G. F., \& Udaeta, M. E. M. (2017). GHG reduction and energy efficiency analyses in a zero-energy solar house archetype. International Journal of Low-Carbon Technologies, 12 (3), 225-232. https://doi.org/10.1093/ijlct/ctx004.

Pelegrino, D. H., Santos, R. G., \& Coelho, R. T. (2019). Experimental evaluation of energy consumption in machine tools: a case study for a two-spindle turning center. Journal of the Brazilian Society of Mechanical Sciences and Engineering. 41(427), 427-436. https://doi.org/10.1007/s40430-019-1921-6.

Portela, T. T., \& Lafay, J. M. S. (2015). Energy Efficiency in Brazil: Policies, motivators, barriers. Renewable Energy and Power Quality Journal, 1(3), 283287. https://doi.org/10.24084/repqj13.305.

Rosa, L. P., \& Lomardo, L. L. B. (2004). The Brazilian energy crisis and a study to support building efficiency legislation. Energy and Buildings, 36(2), 89-95. https://dx.doi.org/10.1016/j.enbuild.2003.09.001.

Santos, A. H. C., Fagá, M. T. W., \& Santos, E. M. (2013). The risks of an energy efficiency policy for buildings based solely on the consumption evaluation of final energy. Electrical Power and Energy Systems, 44(1), 70-77. http://dx.doi.org/10.1016/j.ijepes.2012.07.017.

Scopus. (2020). Guia de referência rápida. https://www.periodicos.capes.gov.br/images/documents/Scopus_Guia\%20de\%20refer\% C3\%AAncia\%20r\%C3\%A1pida_10.08.2016.pdf.

Segundo, A. K. R., Oliveira, D. Filho, Queiroz, J. M., Martins, J. H., \& Monteiro, P. M. B. (2018). Application of methodology for the adequacy of the electrical motor's power sizing: permanent and transient analysis. Mechanic and Energy. 71(2), 197-202. http://dx.doi.org/10.1590/0370-44672015710073.

Silva, F. L. C., Oliveira, F. L. C., \& Souza, R. C. (2019). A bottom-up bayesian extension for long term electricity consumption forecasting. Energy. 167(1), 198-2010. https://doi.org/10.1016/j.energy.2018.10.201.

Silva, F. L. C., Souza, R. C., Oliveira, F. L. C., Lourenco, P. M., \& Calili, R. F. (2018). A bottom-up methodology for long term electricity consumption forecasting of an industrial sector - Application to pulp and paper sector in Brazil. Energy. 144(1), 1107-1118. https://doi.org/10.1016/j.energy.2017.12.078.

Silva, O. A. V. O. L., Santos, F. F. P., Barbosa, F. R., \& Leite, C. S. (2016). Electricity use management based on international protocol: A proposal for UFPI, Brazil. Espacios. 37(11).

Silva, R. D. S, Oliveira, R. C., \& Tostes M. E. L. (2017). Analysis of the Brazilian Energy Efficiency Program for Electricity Distribution Systems. Energies, 1(9), 1-19. https://doi.org/10.3390/en10091391.

Sola, A. V. H., \& Mota, C. M. M. (2015). Melhoria da eficiência energética em sistemas motrizes industriais. Production, 25(3), 498-509. http://dx.doi.org/10.1590/0103-6513.063311.

Sorgato, M. J., Schneider, K., \& Rüther, R. (2018). Technical and economic evaluation of thin-film CdTe building-integrated photovoltaics (BIPV) replacing façade and rooftop materials in office buildings in a warm and sunny climate. Renewable Energy, 118(1), 84-98. https://doi.org/10.1016/j.renene.2017.10.091.

Souza, D. F., Silva P. P. F., Fontenele L. F. A., Barbosa, G. D., \& Jesus M. O. (2019). Efficiency, quality, and environmental impacts: A comparative study of residential artificial lighting. Energy Reports, 5(1), 409-424. https://doi.org/10.1016/j.egyr.2019.03.009.

Souza, R. J., Santos, C. A. C., Ochoa, A. A. V., Marques, A. S., Neto, J. L. M., \& Michima, P. S. A. (2020). Proposal and 3E (energy, exergy, and exergoeconomic) assessment of a cogeneration system using an organic Rankine cycle and an Absorption Refrigeration System in the Northeast Brazil: Thermodynamic investigation of a facility case study. Energy Conversion and Management, 217(1), 1-22. https://doi.org/10.1016/j.enconman.2020.113002.

Viana, C. C. (2019). Estudo da eficiência energética em indústria avícola. [Tese de Mestrado, Universidade Federal da Bahia].

Visentin, C., Trentin, A. W. S., Braun, A. B., \& Thomé, A. (2019). Application of life cycle assessment as a tool for evaluating the sustainability of contaminated sites remediation: A systematic and bibliographic analysis. Science of The Total Environment, 672(1), 893-905. https://doi.org/10.1016/j.scitotenv.2019.04.034.

Visentin, C., Trentin, A. W. S., Braun, A. B., \& Thomé, A. (2020). Life cycle sustainability assessment: A systematic literature review through the application perspective, indicators, and methodologies. Journal of Cleaner Production, 270(1), 1-19. https://doi.org/10.1016/j.jclepro.2020.122509.

Volpi, G., Jannuzzi, G., \& Gomes, R. D. M. (2006). A sustainable electricity blueprint for Brazil. Energy for Sustainable Development, 10(4), 14-24. https://doi.org/10.1016/S0973-0826(08)60552-9.

Wang, L, Wei, Y., \& Brown, M. A. (2017). Global transition to low-carbon electricity: A bibliometric analysis. Applied Energy, 205(1), 57-68. http://dx.doi.org/10.1016/j.apenergy.2017.07.107.

Zanardo, R. P., Siluk, J. C. M., Savian, F. S., \& Schneider P. S. (2018). Energy audit model based on a performance evaluation system. Energy. 154(1), 544552. https://doi.org/10.1016/j.energy.2018.04.179. 